Draft Version August 9, 2018

Preprint typeset using $\mathrm{LAT}_{\mathrm{E}} \mathrm{X}$ style emulateapj v. 08/22/09

\title{
INNER EDGES OF COMPACT DEBRIS DISKS AROUND METAL-RICH WHITE DWARFS.
}

\author{
Roman R. RAFIKov ${ }^{1,2}$ and José A. Garmilla ${ }^{1}$ \\ Draft version August 9, 2018
}

\begin{abstract}
A number of metal-rich white dwarfs (WDs) are known to host compact, dense particle disks, which are thought to be responsible for metal pollution of these stars. In many such systems the inner radii of disks inferred from their spectra are so close to the WD that particles directly exposed to starlight must be heated above $1500 \mathrm{~K}$ and are expected to be unstable against sublimation. To reconcile this expectation with observations we explore particle sublimation in H-poor debris disks around WDs. We show that because of the high metal vapor pressure the characteristic sublimation temperature in these disks is $300-400 \mathrm{~K}$ higher than in their protoplanetary analogues, allowing particles to survive at higher temperatures. We then look at the structure of the inner edges of debris disks and show that they should generically feature superheated inner rims directly exposed to starlight with temperatures reaching $2500-3500 \mathrm{~K}$. Particles migrating through the rim towards the WD (and rapidly sublimating) shield the disk behind them from strong stellar heating, making the survival of solids possible close to the WD. Our model agrees well with observations of WD+disk systems provided that disk particles are composed of Si-rich material such as olivine, and have sizes in the range $\sim 0.03-30 \mathrm{~cm}$.
\end{abstract}

Subject headings: White dwarfs - Accretion, accretion disks - Protoplanetary disks

\section{INTRODUCTION.}

About two dozen (at the moment of writing) white dwarfs (WDs) are known to exhibit near-IR excesses in their spectra (e.g. Zuckerman \& Becklin 1987; Kilic et al. 2005, 2006; Jura et al. 2009, Farihi et al. 2009). This is usually interpreted (Graham et al. 1990; Jura 2003a, 2006; Farihi et al. 2009) as evidence for the existence of nearby solid debris reprocessing stellar radiation in the IR. Detailed spectral modeling of excesses generally supports the idea that debris particles are arranged in a disk-like configuration, which is optically thick but geometrically very thin ${ }^{3}$, thus having properties very similar to the rings of Saturn (Cuzzi et al. 2010). These disks are relatively compact, $\lesssim 1 \mathrm{R}_{\odot}$, excluding the possibility of a primordial origin because of the long cooling ages of the WDs around which they are found, $\sim 0.1-1$ Gyr. It was proposed by Jura (2003b), following an earlier suggestion by Alcock et al. (1986), that these disks are produced by tidal disruption of asteroid-like bodies launched on low-periastron orbits by distant massive planets (Debes \& Sigurdsson 2002), which have survived the asymptotic giant branch (AGB) phase of the evolution of the central star.

All WDs possessing compact debris disks exhibit atmospheres which are polluted (sometimes heavily) with metals, putting these WDs into the DAZ and DBZ classes (Farihi 2011). This observation strongly suggests that many (if not all) metal-rich WDs are polluted by accretion of high- $\mathrm{Z}$ elements from the compact debris disks around them. In cases where near-IR observations do not reveal the presence of a conspicuous disk of solids,

\footnotetext{
${ }^{1}$ Department of Astrophysical Sciences, Princeton University, Ivy Lane, Princeton, NJ 08540; rrr,garmilla@astro.princeton.edu

2 Sloan Fellow

3 Some exceptions are also known such as HD233517, the spectrum of which is better fitted by invoking a flared disk (Jura 2003a), or GD56, which is better fitted by a warped disk (Jura et al.|2009).
}

the disk may simply be too tenuous to reprocess enough stellar radiation to make itself visible. Alternatively, the disk of solids may have dispersed some time ago but the metals can still be present in the WD atmosphere because of their long settling time (Metzger et al. 2012).

In this scenario of WD metal pollution (which is certainly valid for systems with known disks) the issue of metal transfer onto the WD surface must be addressed, as the disk of solids cannot extend inward all the way to the stellar surface at $R_{\star}$. Because of the high effective temperature of these WDs, $T_{\star} \sim(7-20) \times 10^{3} \mathrm{~K}$, solids must sublimate at some inner radius $R_{i n}$ producing metal gas, which is subsequently accreted onto the WD via a conventional accretion disk. The existence of inner cavities in disks of solid debris follows directly from the shape of their spectral energy distributions (SEDs), which generally show a lack of emission corresponding to temperatures in excess of $\sim 2000 \mathrm{~K}$.

It has been shown by Rafikov (2011a) and Bochkarev \& Rafikov (2011) that Poynting-Robertson (PR)drag on the disk of particles naturally drives accretion of solids at rates $\dot{M}_{Z} \sim 10^{7}-10^{8} \mathrm{~g} \mathrm{~s}^{-1}$. Their sublimation feeds metal gas accretion onto the WD surface at the same rate. Even higher $\dot{M}_{Z}$ can be achieved if the disk of solids can couple via aerodynamic drag to the surrounding gaseous disk (Gänsicke et al. 2006, 2007; Brinkworth et al. 2009; Melis et al. 2010), which naturally forms via sublimation of particles at $R_{\text {in }}$ (Rafikov 2011b; Metzger et al. 2012).

\subsection{Inner rim puzzle}

Existing debris disk models used to fit SEDs try to link the radius of the inner edge of the disk $R_{i n}$ to a certain value of the "sublimation temperature" $T_{s}$, which depends on physical properties of the constituent particles. In these models particles sublimate at radii where their temperature $T$ exceeds the sublimation temperature $T_{s}$, 
and the inner radius $R_{i n}$ corresponds to $T\left(R_{i n}\right)=T_{s}$. Then the determination of $R_{i n}$ hinges upon the proper choice of $T_{s}$ and the knowledge of a relation between $T$ and $r$.

The characteristic value of $T_{s}$ usually assumed for disks around WDs is $1300-1500 \mathrm{~K}$. This is a typical sublimation temperature for the Si-rich solids, such as olivine or calcium-aluminum inclusions (CAIs) based on Lodders (2003) who calculated condensation temperatures of different species in the proto-Solar disk assuming Solar abundances of elements. There is good evidence that Si-rich material indeed represents a significant fraction of mass in the debris disks around WDs, both from the detections of the Si feature at $10 \mu \mathrm{m}$ in spectra of such disks (Jura et al. 2009) and the atmospheric compositions of their host WDs (Zuckerman et al. 2007; Klein et al. 2010, 2011; Jura et al. 2012). However, as we show in $\$ 2$ this estimate of $T_{s}$ needs to be seriously revised for the typical conditions in circum-WD debris disks.

There is certain ambiguity regarding the equilibrium temperature of the disk particles. In Appendix $\mathrm{A}$ we consider their thermal balance by looking at different heating and cooling processes, and find stellar heating and radiative cooling of particles to dominate the balance. In this case particles directly exposed to starlight or located in the optically thin parts of the disk, are heated to a temperature

$$
T_{\text {thin }}(r) \approx T_{\star}\left(\frac{R_{\star}}{2 r}\right)^{1 / 2} .
$$

In particular, this estimate is appropriate for particles at the inner edge of the optically thick disk because these are directly illuminated by the star. However, behind the narrow rim of directly exposed particles the disk is illuminated by starlight only at its surface at a grazing incidence angle $\zeta \approx(4 / 3 \pi) R_{\star} / r$ (Friedjung 1985) for a geometrically thin (i.e. flat) disk. This is because of the shielding that is provided by the rim particles against direct starlight for the disk just outside the rim, see Figure 1 for illustration. The equilibrium temperature of particles in the optically thick parts of the disk is given by (Chiang \& Goldreich 1997)

$$
T_{\text {thick }}(r)=T_{\star}\left(\frac{2}{3 \pi}\right)^{1 / 4}\left(\frac{R_{\star}}{r}\right)^{3 / 4} .
$$

This expression is valid in the shielded parts of the disk where the near-IR emission is produced.

The assumption that particles sublimate at a single temperature $T_{s}$ implies that directly exposed rim particles cannot be hotter than $T_{s}$ (Rafikov 2011a, b). However, using equation (11) to estimate $T(r)$ at the inner edge of the disk and taking $T_{s} \approx 1500 \mathrm{~K}$ one finds that the near-IR contribution to the SED produced by the disk is too weak to account for observations. Indeed, combining equations (11) and (2) one finds

$$
T_{\text {thick }}=\left(\frac{16}{3 \pi}\right)^{1 / 4} T_{\text {thin }}\left(\frac{T_{\text {thin }}}{T_{\star}}\right)^{1 / 2} .
$$

Thus, when $T_{\text {thin }}$ is close to the sublimation temperature the temperature $T_{\text {thick }}$ in the shielded part of the disk must be substantially lower than $T_{s}$ (since necessarily $\left.T_{\text {thin }}<T_{\star}\right)$. For example, taking $T_{\star}=10^{4} \mathrm{~K}$ and $T_{\text {thin }}\left(R_{\text {in }}\right)=T_{s}=1500 \mathrm{~K}$ one finds $T_{\text {thick }}\left(R_{\text {in }}\right) \approx 660 \mathrm{~K}$. As a result, the SED of such a disk is going to be very deficient of the near-IR flux corresponding to emission at temperatures $\gtrsim 1300-1500 \mathrm{~K}$. However, disk SEDs typically exhibit considerable emission by material heated in excess of $1000 \mathrm{~K}$, see Table 2. This is hard to reconcile with only the inner rim of the disk being heated to $T_{s}$.

Jura \& Xu (2012) suggested that this problem can be resolved if the inner edge of the disk is set by sublimation occurring in the optically thick part of the disk illuminated by the star at grazing incidence, rather than in the thin inner rim of directly exposed particles. This is equivalent to determining the value of $R_{i n}$ by using the expression (2) instead of (11) in equation $T\left(R_{i n}\right)=T_{s}$. If that were true, however, then the temperature at the inner rim must be higher than $T_{s}$, see equation (3), and rim particles would be sublimating, exposing the particles behind them to direct starlight. As a result, the inner rim would recede to larger distance from the WD until it reaches the radius where $T_{\text {thin }}=T_{s}$, so we go back to the previously considered situation with its intrinsic problems. Only this configuration is going to be in stable phase equilibrium as long as sublimation is idealized as a step-like process, i.e. that particles turn into gas as soon as they reach $T_{s}$. Such an equilibrium was assumed in Rafikov $(2011 \mathrm{a}, \mathrm{b})$ to determine $R_{i n}$.

This set of conflicting arguments suggests that our understanding of the location and structure of the inner rim is in some ways incomplete. The goal of the present work is to fill these gaps and to provide a more detailed picture of the sublimation of solids at the inner edge of the disk by focussing on two effects. First, in 92 we show that sublimation in hydrogen-poor debris disks around WDs is different from sublimation in the proto-Solar disk resulting in $T_{s}$ being higher than $1500 \mathrm{~K}$. Second, we show in 33 that particle sublimation at the inner rim of an optically thick disk is a dynamic process, which makes it possible for the rim particles to reach temperatures in excess of $T_{s}$ before sublimating. In $\$ 4$ we look at sublimation in optically thin disks. We apply our theory to observed disk-hosting WDs in 45 , and discuss our findings in 6. A summary of our main results can be found in 97.

\section{SPECIFICS OF SUBLIMATION IN THE CIRCUM-WD DISKS.}

Solid particles of certain composition surrounded by vapor with the same elemental abundance grow by condensation of molecules or atoms arriving at their surfaces from the gas phase and lose mass due to sublimation. For a particle of mass $m_{p}$ and surface area $S_{p}$ surrounded by vapor at pressure $P_{\text {vap }}$ one can write the following mass evolution equation (Guhathakurta \& Draine 1989):

$$
\frac{d m_{p}}{d t}=S_{p}\left[\langle\alpha\rangle P_{\operatorname{vap}}\left(\frac{\mu}{2 \pi k_{B} T}\right)^{1 / 2}-\dot{m}(T)\right] .
$$

where $\mu$ is the mean molecular weight of the particle material. Here the first term in brackets describes condensation $(\langle\alpha\rangle$ is the accommodation coefficient — sticking probability of gas particles impacting the solid surface), 
while the second term characterizes sublimation from the particle surface ( $\dot{m}$ is the mass loss rate per unit surface area due to sublimation).

When the vapor pressure becomes equal to the saturated vapor pressure $P_{\text {vap }}^{\text {sat }}(T)$ at a given temperature $T$, the equilibrium between the loss and gain processes is established and $d m_{p} / d t=0$. This allows us to express

$$
\dot{m}(T)=\langle\alpha\rangle P_{\mathrm{vap}}^{\mathrm{sat}}(T)\left(\frac{\mu}{2 \pi k_{B} T}\right)^{1 / 2} .
$$

The concept of sublimation temperature $T_{s}$ implies the process of phase transition from solid to gas to occur in a step-like fashion. At $T=T_{s}$ the vapor saturates and an infinitesimal increase of temperature leads to slow (quasistatic) conversion of solid into gas. Then one can again assume $d m_{p} / d t \rightarrow 0$ and the right-hand side of equation (41) then provides us with an implicit relation for $T_{s}$ as a function of the vapor pressure $P_{\text {vap }}$, as long as the dependence $\dot{m}(T)$ is known.

Considerations based on the Clausius-Clapeyron relation suggest that

$$
P_{\mathrm{vap}}^{\mathrm{sat}}(T) \propto T^{\beta} e^{-T_{0} / T},
$$

where the constants $\beta$ and $T_{0}$ are specific to a particular particle composition. Since the strongest dependence of $P_{\text {vap }}^{\text {sat }}$ on $T$ occurs through the exponential factor, the power-law dependence on $T$ in equation (51) can be absorbed into the (approximately) constant pre-factor, so that $\dot{m}(T)$ is approximated as

$$
\dot{m}(T)=\langle\alpha\rangle K_{0} e^{-T_{0} / T},
$$

where $K_{0}$ is a constant. This is the form of $\dot{m}(T)$ that we adopt in this work.

In the following we will consider a number of different materials which can represent the composition of disk particles. We summarize the parameters $K_{0}$ and $T_{0}$ for different species considered in this work in Table 1, and provide details of their calculation in Appendix B

Setting the left hand side of equation (44) to zero and using $\dot{m}$ in the form (7) we obtain the (implicit) dependence of $T_{s}$ on the vapor pressure:

$$
\begin{aligned}
T_{s}\left(P_{\text {vap }}\right) & =T_{0}\left(\ln \Lambda_{s}\right)^{-1}, \\
\Lambda_{s} & =\frac{K_{0}}{P_{\text {vap }}}\left(\frac{2 \pi k T_{s}}{\mu}\right)^{1 / 2},
\end{aligned}
$$

with $\Lambda_{s} \gg 1$. According to this expression $T_{s}$ is higher for larger $P_{\text {vap }}$, even though the dependence is rather weak (logarithmic).

The vapor pressure in the gas around the rim can be easily estimated if the disks consist of particles with identical composition. In general this does not have to be true but we still adopt this assumption for simplicity. Then metals detected in the WD atmosphere come from accretion of this material in the gas phase, and the measurement of the corresponding mass accretion rate

$$
\dot{M}_{Z}=3 \pi \nu \Sigma_{g}
$$

(where $\nu=\alpha_{\nu} c_{s}^{2} / \Omega$ is the kinematic viscosity, $\alpha_{\nu}$ is the effective viscosity parameter, $\Omega$ is the Keplerian angular frequency, and $c_{s}$ and $\Sigma_{g}$ are the sound speed and the
Table 1

Sublimation properties of different materials

\begin{tabular}{lcccc}
\hline \hline Material & $\begin{array}{c}K_{0} \\
\mathrm{~g}^{-1} \mathrm{~cm}^{-2} \mathrm{~s}^{-1}\end{array}$ & $\begin{array}{c}T_{0} \\
\mathrm{~K}\end{array}$ & $\begin{array}{c}\mu \\
\mathrm{m}_{\mathrm{p}}\end{array}$ & $\begin{array}{c}T_{\text {sub }}\left(1 \text { dyne } \mathrm{cm}^{-2}\right) \\
\mathrm{K}\end{array}$ \\
\hline Olivine & $1.6 \times 10^{9}$ & 68100 & 141 & 2100 \\
Graphite & $9.2 \times 10^{7}$ & 81200 & 12 & 2600 \\
$\mathrm{CAI}$ & $1.1 \times 10^{10}$ & 69400 & 274 & 2000 \\
Iron & $2.3 \times 10^{7}$ & 45400 & 56 & 1600 \\
$\mathrm{Al}_{2} \mathrm{O}_{3}$ & $8 \times 10^{9}$ & 80500 & 102 & 2300 \\
$\mathrm{SiC}$ & $6 \times 10^{8}$ & 73700 & 40 & 2300 \\
& & & & \\
\hline
\end{tabular}

surface density of the gas) provides an estimate of the vapor pressure:

$$
\begin{aligned}
P_{\text {vap }} & \approx \frac{\dot{M}_{Z} \Omega^{2}}{3 \pi \alpha_{\nu} c_{s}} \\
& \approx 0.5 \text { dyne } \mathrm{cm}^{-2} \frac{\dot{M}_{Z, 8} M_{\star, 1}}{\alpha_{\nu,-2} c_{s, 1}}\left(\frac{0.2 R_{\odot}}{r}\right)^{3} .
\end{aligned}
$$

Here $\dot{M}_{Z, 8} \equiv \dot{M}_{Z} /\left(10^{8} \mathrm{~g} \mathrm{~s}^{-1}\right), M_{\star, 1} \equiv M_{\star} / M_{\odot}, \alpha_{\nu,-2} \equiv$ $\alpha_{\nu} / 10^{-2}$, and $c_{s, 1} \equiv c_{s} /\left(1 \mathrm{~km} \mathrm{~s}^{-1}\right)$ is the characteristic value of the sound speed for Si-rich material heated to temperature of several $10^{3} \mathrm{~K}$. According to equation (11) $P_{\text {vap }} \sim 1$ dyne $\mathrm{cm}^{-2}$ for $\dot{M}_{Z} \sim 10^{8} \mathrm{~g} \mathrm{~s}^{-1}$, which is a characteristic mass accretion rate of metals due to the $\mathrm{PR}$ drag (Rafikov 2011a). It should also be mentioned that a number of metal-rich. WDs with debris disks exhibit much higher values of $\dot{M}_{Z}$, easily reaching $10^{9}-10^{10} \mathrm{~g}$ $\mathrm{s}^{-1}$. In these systems $P_{\text {vap }} \sim 10-100$ dyne $\mathrm{cm}^{-2}$ should be typical.

In Table 1 we show the values of $T_{s}$ computed from equation (8) for different materials assuming $P_{\text {vap }}=1$ dyne $\mathrm{cm}^{-2}$. One can see that these values are considerably higher than the conventional estimate $T_{s} \sim 1500$ $\mathrm{K}$ often used in modeling debris disk SEDs. The explanation for this puzzling difference lies in the fact that the canonical estimate is based on the work of Lodders (2003) which explicitly assumes a Solar composition gas in equilibrium with sublimating particles to compute $T_{s}$. Even though a total pressure of 100 dyne $\mathrm{cm}^{-2}$ is assumed in that work the vapor pressure $P_{\text {vap }}$ of high- $\mathrm{Z}$ species is going to be much lower in protoplanetary disks because of the low abundance of such elements compared to $\mathrm{H}$, which contributes most to the total pressure. For example, in Lodders (2003) iron was assumed to have an abundance (by number, with respect to $\mathrm{H}$ ) of $3.4 \times 10^{-5}$, which results in vapor pressure of atomic $\mathrm{Fe}$ of $3.4 \times 10^{-3}$ dyne $\mathrm{cm}^{-2}$ (assuming that molecular $\mathrm{H}$ has dissociated and the total pressure in the gas is $100 \mathrm{dyne} \mathrm{cm}^{-2}$ ). Using equation (8) we then find $T_{s} \approx 1300 \mathrm{~K}$ instead of $1600 \mathrm{~K}$ typical for a debris disk around a WD, if the latter were composed of pure $\mathrm{Fe}$ and had a total pressure (equal to the Fe vapor pressure) of 1 dyne $\mathrm{cm}^{-2}$. A similar or even larger difference with the canonical estimate of $T_{s}$ arises for other elements listed in Table 1.

A deficiency of volatile components $(\mathrm{H}$ and $\mathrm{He})$ and high relative abundance of metals (can easily be as high 
as unity) in the gaseous phase of the debris disks around WDs naturally results in high values of the (quasi-static) sublimation temperature $T_{s}$ in these systems. This goes in the direction of alleviating the puzzle of high temperatures of solid particles inferred from the SED modeling for these objects. However, it does not fully resolve this problem because in the simple model of sublimation $T_{s}$ is still reached only in the inner rim of the disk, i.e. $T_{s}=T_{\text {thin }}\left(r_{\text {rim }}\right)$. The temperature in the bulk of the disk just behind the rim is again much lower than $T_{s}$ : for $T_{\star}=10^{4} \mathrm{~K}$ and $T_{s}=2100 \mathrm{~K}$ as typical for olivines (see Table 10) one finds using equation (3) that $T_{\text {thick }}\left(r_{\text {rim }}\right) \approx 1100 \mathrm{~K}$, which is clearly not enough to reproduce the short-wavelength portion of the observed SED in many WD+disk systems, see Table 2.

In the following section we provide a complete solution to this puzzle by considering particle sublimation in more detail.

\section{INNER EDGE STRUCTURE IN THE OPTICALLY THICK DISK}

We now present a simple physical model for the structure of the inner rim of the disk, the inner part of which is optically thick, see Figure 1] the vertical optical depth of such a disk

$$
\tau \equiv \frac{3}{4} \frac{\Sigma}{\rho a}
$$

( $a$ and $\rho$ are the particle radius and bulk density, $\Sigma$ is the surface density of the disk of solids) is larger than unity. This implies that the disk absorbs all incident stellar radiation because its optical depth to starlight (Rafikov 2011a) $\tau_{\|} \equiv \tau / \zeta \gg 1$. According to Rafikov (2011b) and Metzger et al. (2012) such optically thick inner regions are natural for massive debris disks in which aerodynamic coupling to the surrounding gaseous disk is strong enough to drive runaway accretion of metals onto the WD. In this case the particle surface density at $R_{\text {in }}$ is high enough for the inner disk to stay optically thick.

The key ingredients of our model are

- the inward migration of particles across the rim region,

- the shielding from starlight provided by the directly exposed rim particles to particles further out,

- the dynamic regime of particle sublimation, as opposed to the quasi-static situation explored previously.

Our primary goal here is to determine the temperature inside the rim $T_{\text {rim }}$ and the distance $R_{i n}$ at which the disk gets truncated by sublimation.

In the previous section, we assumed that particle sublimation occurs at a single temperature $T_{s}$, so that particles cannot exist in solid form at $T>T_{s}$. This, however, is not true on time intervals shorter than the time it takes to completely sublimate a particle. Using equation (4) one can estimate the instantaneous sublimation timescale as the time it takes to completely sublimate a particle (neglecting condensation) at a given temperature

$$
t_{s}(T) \equiv \frac{m_{p}}{S_{p} \dot{m}(T)} .
$$

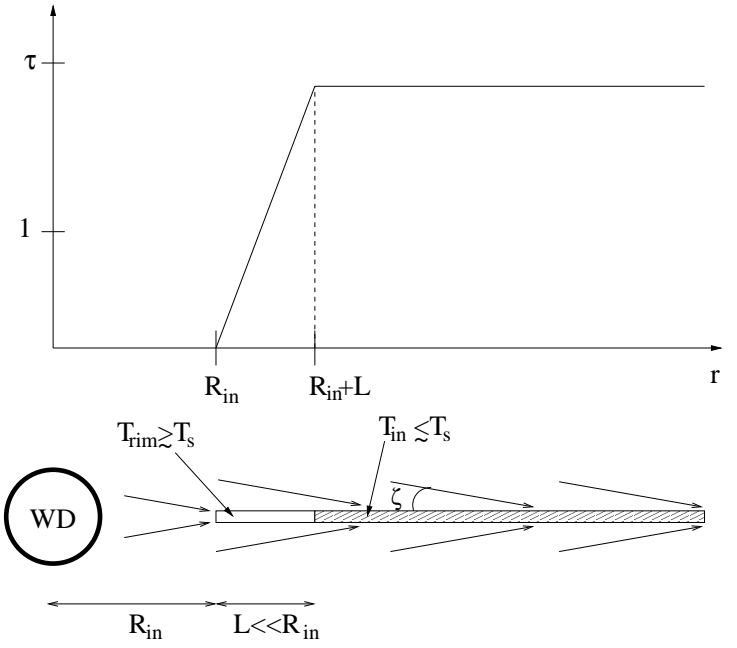

Figure 1. Schematic representation of the inner rim structure and surface density distribution in its vicinity in the optically thick $(\tau \gtrsim 1)$ case. The shaded part of the disk heated to $T_{i n} \lesssim T_{s}$ receives starlight only at the surface, at grazing incidence angle $\zeta$. Particles in the inner, unshaded part are directly illuminated by the star and are heated to $T_{\text {rim }} \gtrsim T_{s}$. Radial width $L$ of this exposed rim is determined by equation (16).

Sublimation should be considered as a dynamic (as opposed to quasi-static, like in the previous section) process whenever the particle temperature changes on a time scale $\lesssim t_{s}\left(T_{s}\right)$

When the temperature of a solid object is close to its sublimation temperature $T_{s}\left(P_{\text {vap }}\right)$ (for a given vapor pressure $P_{\text {vap }}$, which at $T_{s}$ should be equal to $\left.P_{\text {vap }}^{\text {sat }}\right)$ equations (5), (4) and (13) allow us to estimate

$$
\begin{aligned}
t_{s}\left(T_{s}\right) & \approx \frac{a_{0} \rho}{3\langle\alpha\rangle P_{\text {vap }}}\left(\frac{2 \pi k_{B} T}{\mu}\right)^{1 / 2} \\
& \approx 7 \mathrm{~d} \frac{a_{0,1} P_{1} \rho_{1}}{\langle\alpha\rangle_{0.1} \mu_{28}^{1 / 2}}\left(\frac{T_{s}}{2000 \mathrm{~K}}\right)^{1 / 2}
\end{aligned}
$$

(for a spherical particle of initial radius $a_{0}$ ) where $a_{0,1} \equiv$

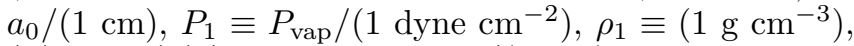
$\langle\alpha\rangle_{0.1} \equiv\langle\alpha\rangle / 0.1$, and $\mu_{28} \equiv \mu /\left(28 m_{p}\right)$, as appropriate for Si. Because of the rapid scaling of $\dot{m}(T)$ with $T$ it is obvious that $t_{s}(T)$ is a very sensitive function of $T$, and $t_{s}(T) \ll t_{s}\left(T_{s}\right)$ even if $T$ is just slightly higher than $T_{s}$.

As mentioned in 1.1 in the optically thick disk particles just outside the rim are shielded from direct starlight by the rim particles and their temperature is given by $T_{i n}=T_{\text {thick }}\left(R_{i n}\right)$, see equation (2) and Figure [1] This is the highest temperature that one would infer from fitting the flat optically-thick disk model to the SED. These shielded particles are cool enough for sublimation not to be important - an assumption that we check later.

Particles in the disk migrate inwards due to PR drag or aerodynamic coupling to the gaseous disk - this migration is what ultimately gives rise to metal accretion onto the WD. As particles enter the rim and get exposed to direct starlight their temperature rapidly goes up to $T_{\text {rim }}=T_{\text {thin }}\left(R_{\text {in }}\right) \gtrsim T_{\text {in }}$ (the amount of energy required to heat the particle by several hundred $K$ is small compared to the heat of sublimation). According to equation 
(1) the inner rim of the optically thick disk lies at

$$
R_{\text {in }}^{\text {thick }}=\frac{R_{\star}}{2}\left(\frac{T_{\star}}{T_{\text {rim }}}\right)^{2} .
$$

The fact that particles in the rim are illuminated by virtually unattenuated stellar radiation implies that the optical depth of the rim to starlight in the radial direction

$$
\tau_{\|} \approx \int_{0}^{L} n(x) \times \pi a^{2}(x) d x \approx 1,
$$

where $L$ is the radial extent of the rim, $x$ is the radial distance away from the inner edge of the rim (i.e. the location where all particles sublimate; rim corresponds to $0<x<L), n(x)$ is the volume number density of particles, and we assume the particle cross section for starlight to be equal to the geometric cross section $\pi a^{2}$ (assuming spherical particles).

Since solids lose mass to sublimation while drifting through the rim, particle radius $a$ is a function of $x$; in particular $a(x=0)=0$. The evolution of particle size due to sublimation is described by the following simple equation

$$
\frac{d}{d t}\left(\frac{4 \pi}{3} \rho a^{3}\right) \approx-4 \pi a^{2} \dot{m}\left(T_{\text {rim }}\right),
$$

which is a simplified version of equation (4) in which condensation has been neglected. This is a reasonable assumption because we will find later that the rim temperature $T_{\text {rim }}$ is significantly higher than the quasi-static sublimation temperature $T_{s}\left(P_{\text {vap }}\right)$. In this case the flux of molecules (or atoms) leaving the particle surface is much higher than the flux of particles arriving at it (for a given surrounding vapor pressure $P_{\text {vap }}$ ), so that condensation can be neglected.

We assume that the disk outside the rim is composed of particles of a single size $a_{0}$ so that $a(x=L)=a_{0}$. Introducing $v_{r} \equiv d r / d t=d x / d t$ one can write $d a / d t=$ $v_{r} d a / d x$, so that equation (17) reduces to

$$
\frac{d a}{d x}=\frac{\dot{m}\left(T_{\text {rim }}\right)}{\rho v_{r}(a, x)} .
$$

In general $v_{r}(a, x)$ is a function of both $a$ and $x$, see e.g. equation (31) for the case of PR drag-driven accretion.

We will now assume that as particles pass through the rim and sublimate, their number flux $F_{N}$ does not change (until they fully sublimate) even though their mass flux varies because their sizes go down as a result of sublimation. This assumption amounts to neglecting the possibility of particle breaking or merging during their travel through the rim.

Introducing the vertical thickness of the disk $h(x)$ one can use the constancy of $F_{N}$ to express volume number density of particles in the rim $n(x)$ as

$$
n(x)=\frac{F_{N}}{2 \pi R_{i n}} \times \frac{1}{v_{r}(x) h(x)} .
$$

We can now express $v_{r}$ from equation (18), plug it into equation (19) and substitute the resulting expression for

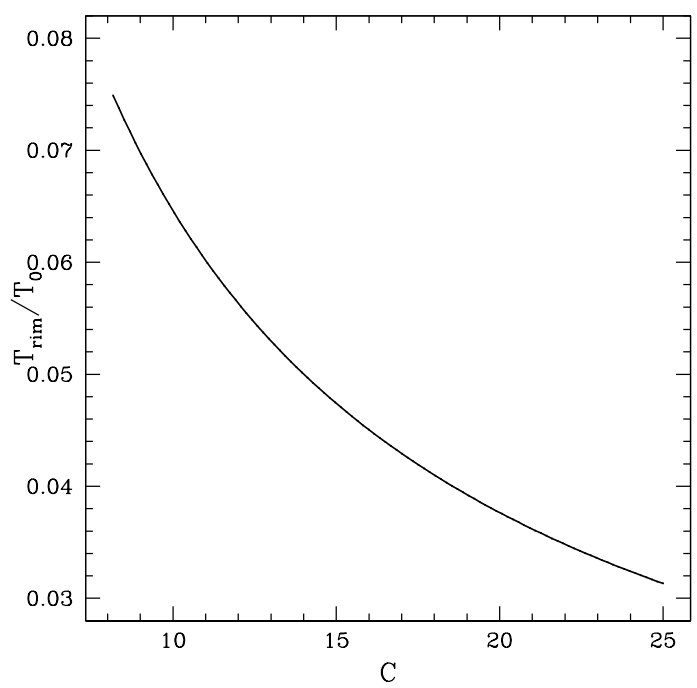

Figure 2. Solution of equation (28) for the scaled temperature of the inner $\operatorname{rim} T_{\text {rim }} / T_{0}$ as a function of the dimensionless parameter $C$ given by equation (29) which contains all information about the parameters of the system and particle properties.

$n(x)$ into the condition (16) to find that

$$
\frac{F_{N} \rho}{2 R_{\text {in }} \dot{m}\left(T_{\text {rim }}\right)} \int_{0}^{L} \frac{a^{2}}{h(x)} \frac{d a}{d x} d x \approx 1 .
$$

To proceed further we need to make explicit assumptions regarding the behavior of $h(x)$. Debris disks around WDs are expected to be similar in properties to dense planetary rings around Saturn. The latter have vertical thickness comparable to the particle size, which is established by collisions between particles. Thus, it may be natural to assume that $h(x) \sim a(x)$, which upon plugging into equation (20) and integrating with the condition $a(L)=a_{0}$ gives

$$
\frac{F_{N} \rho a_{0}^{2}}{4 R_{\text {in }} \dot{m}\left(T_{\text {rim }}\right)} \approx 1 .
$$

The mass accretion rate of metals onto the WD $\dot{M}_{Z}$ is related to $F_{N}$ via $\dot{M}_{Z}=F_{N} \times(4 \pi / 3) \rho a_{0}^{3}$, so that equation (21) ultimately yields

$$
\dot{m}\left(T_{\text {rim }}\right) \approx \frac{3}{8} \frac{\dot{M}_{Z}}{2 \pi R_{\text {in }} a_{0}} .
$$

One can try another simple approximation for the behavior of $h(x)$, namely assuming that $h \sim a_{0}=$ const. In this case one again recovers condition (22) with a factor of $1 / 4$ instead of $3 / 8$. This similarity of results suggests that, for any reasonable assumption regarding the behavior of $h(x)$, the condition

$$
\dot{m}\left(T_{\text {rim }}\right) \approx \zeta \frac{\dot{M}_{Z}}{R_{\text {in }} a_{0}},
$$

with $\zeta \sim 0.05-0.1$ must be satisfied in the rim.

Equation (23) is the condition that determines the value of the inner rim temperature $T_{\text {rim }}$ (or, equivalently, the inner radius $R_{i n}$ ) once the explicit form of $\dot{m}\left(T_{\text {rim }}\right)$ is specified. Given that $2 \pi R_{i n} a_{0}$ is the area of the inner 
rim as seen from the WD, equation (23) suggests a simple physical interpretation: the disk is truncated at the distance $R_{i n}$, where the full rate of sublimation from the area of its inner rim facing the star $\left(\sim 2 \pi R_{i n} a_{0}\right)$ roughly matches the metal accretion rate through the disk $\dot{M}_{Z}$.

By taking $d a \sim a_{0}, d x \sim L$ in equation (18) we estimate the time $t_{\text {cross }}$ it takes particles to cross the rim (and sublimate): $t_{\text {cross }} \sim L / v_{r} \sim \rho a_{0} / \dot{m}\left(T_{\text {rim }}\right)$. Using equation (23) to express $\dot{m}\left(T_{\text {rim }}\right)$ via $\dot{M}_{Z}$ and equation (15) for $R_{\text {in }}$ we obtain

$$
\begin{aligned}
t_{\text {cross }} & \sim \frac{\rho a_{0}^{2} R_{\star}}{2 \zeta \dot{M}_{Z}}\left(\frac{T_{\star}}{T_{\text {rim }}}\right)^{2} \\
& \approx 400 \mathrm{~s} \frac{\rho_{1} a_{0,1}^{2} R_{\star,-2}}{\zeta_{0.1} \dot{M}_{Z, 8}}\left(\frac{T_{\star} / T_{\text {rim }}}{3}\right)^{2},
\end{aligned}
$$

where $R_{\star,-2} \equiv R_{\star} / 10^{-2} R_{\odot}, \zeta_{0.1} \equiv \zeta / 0.1$. Interestingly, this estimate is independent of the nature of the physical process driving particle migration.

We now plug $R_{i n}$ expressed in terms of $T_{\text {rim }}$ via equation (15) into equation (23) to find the following transcendental equation for $T_{\text {rim }}$ only:

$$
\langle\alpha\rangle K_{0} e^{-T_{0} / T_{\mathrm{rim}}} \approx 2 \zeta \frac{\dot{M}_{Z}}{R_{\star} a_{0}}\left(\frac{T_{\mathrm{rim}}}{T_{\star}}\right)^{2},
$$

from which we find

$$
\begin{aligned}
& \frac{T_{\text {rim }}}{T_{0}}=\left(\ln \Lambda_{\text {rim }}\right)^{-1}, \\
& \Lambda_{\text {rim }}=\frac{\langle\alpha\rangle K_{0} R_{\star} a_{0}}{2 \zeta \dot{M}_{Z}}\left(\frac{T_{\star}}{T_{\text {rim }}}\right)^{2},
\end{aligned}
$$

with $\Lambda_{\text {rim }} \gg 1$. This result can be re-written in the following simple form amenable for iterative solution:

$$
\begin{aligned}
\frac{T_{\text {rim }}}{T_{0}} & =\left[C-2 \ln \frac{T_{\text {rim }}}{T_{0}}\right]^{-1}, \\
C & =\ln \left[\frac{\langle\alpha\rangle K_{0} R_{\star} a_{0}}{2 \zeta \dot{M}_{Z}}\left(\frac{T_{\star}}{T_{0}}\right)^{2}\right] \\
& \approx 18.6+\ln \frac{\langle\alpha\rangle_{0.1} R_{\star,-2} a_{0,1} T_{\star, 4}^{2}}{\zeta_{0.1} \dot{M}_{Z, 8}}
\end{aligned}
$$

where $T_{\star, 4} \equiv T_{\star} /\left(10^{4} \mathrm{~K}\right)$ and the numerical estimate in equation (29) is done for olivine $\left(K_{0}=1.6 \times 10^{9} \mathrm{~g}^{-1}\right.$ $\mathrm{cm}^{-2} \mathrm{~s}^{-1}, T_{0}=68,100 \mathrm{~K}$, see Table 1). Figure 2 shows the exact solution of equation (26) for $T_{\text {rim }} / T_{0}$ as a function of $C$. This curve is independent of the system parameters and particle properties $\left(\dot{M}_{Z}, a_{0}\right.$, composition), which are all absorbed into the definition of $C$.

Using equations (8), (11) and (24) it can be trivially shown that

$$
\frac{\Lambda_{s}}{\Lambda_{\text {rim }}}=\frac{t_{s}}{t_{\text {cross }}} .
$$

As a result, when the time $t_{\text {cross }}$ it takes for a particle to cross the rim is shorter than the sublimation timescale $t_{s}$ one finds that $\Lambda_{s} \gg \Lambda_{\text {rim }}$ and $T_{\text {rim }} \gtrsim T_{s}$. This illustrates our expectation that in the case of dynamical sublimation the temperature of particles can be higher than the quasistatic sublimation temperature $T_{s}$ given by equation (8).
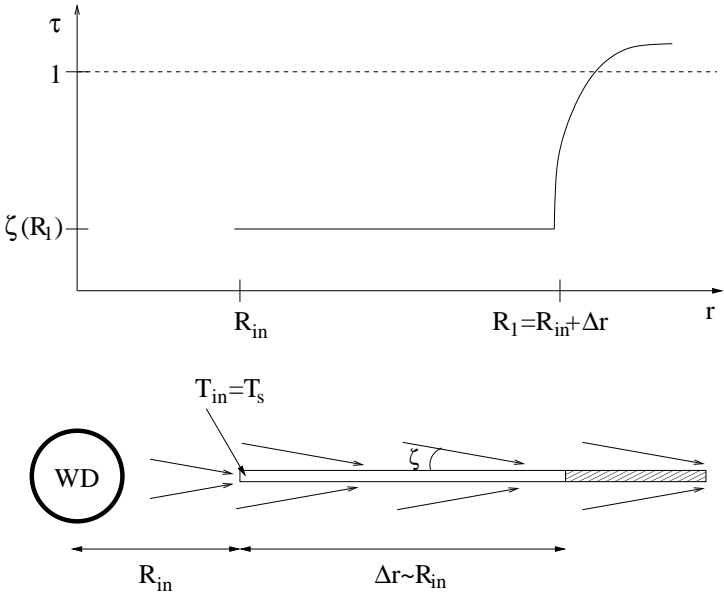

Figure 3. Schematic representation of the inner rim structure and the surface density distribution in its vicinity in the optically thin $\left(\tau_{\|} \lesssim 1\right)$ inner disk. The inner optically thin part of the disk (unshaded) is directly illuminated by the star over a broad range of radii $\Delta r \sim R_{i n}$. The optically thick part (shaded) starts at $R_{1}=R_{i n}+\Delta r$. Compare with Figure 1

For example, for the fiducial values of parameters adopted in equation (29) one finds for olivine $C=18.6$ and $T_{\text {rim }} \approx 0.04 T_{0} \approx 2700 \mathrm{~K}$, while according to Table 1 olivine has $T_{s} \approx 2100$. The inner edge of the disk in this case is located very close to the WD surface, at $R_{\text {in }} \approx 7 R_{\star}$, see equation (15).

At the same time, just behind the rim the disk temperature is $T_{\text {in }}=T_{\text {thick }}\left(R_{\text {in }}\right) \approx 1600 \mathrm{~K}<T_{s}$ for $T_{\star}=10^{4} \mathrm{~K}$, see equation (31). This verifies our previous assumption of relatively low particle temperature (i.e. $T_{i n}<T_{s}$ ) just behind the rim, justifying the disregard of particle sublimation in this region. On the other hand, this value of $T_{i n}$ is clearly high enough for the disk to produce enough near-IR emission corresponding to $T \sim 1500 \mathrm{~K}$, in agreement with observations.

Equation (30) also emphasizes the necessity of particle accretion for maintaining the superheated inner rim: if $\dot{M}_{Z} \rightarrow 0$ then according to equation (24) $t_{\text {cross }} \rightarrow \infty$ and the hot inner rim does not exist.

\section{SUBLIMATION RADII IN DISKS WITH OPTICALLY THIN $\left(\tau_{\|} \lesssim 1\right)$ INNER REGIONS}

We now look at the case of a disk, the inner part of which is optically thin for incident stellar radiation, i.e. $\tau_{\|} \lesssim 1$, see Figure 3 . As demonstrated by Bochkarev \& Rafikov (2011) such situation naturally arises for a low mass disk, which starts with $\tau_{\|} \lesssim 1$ everywhere, or for a moderately massive disk, which hasn't gone through the runaway accretion phase. In the latter case, as shown in Bochkarev \& Rafikov (2011), an optically thin tail of solid material with $\tau_{\|} \sim 1$ (or $\tau \sim \zeta \ll 1$ ) naturally develops as an inward extension of the optically thick part of the disk under the action of the PR drag.

Particles in such optically thin tail are directly exposed to starlight, meaning that their equilibrium temperature is given by equation (11). Also, Rafikov (2011b) and Metzger et al. (2012) have shown that the dynamics of these optically thin regions, including the radial drift of particles, is determined primarily by PR drag. Then the 
radial migration speed is just

$$
v_{r, P R}=\frac{3}{8 \pi} \frac{L_{\star}}{\rho a_{0} c^{2}} \frac{1}{r} .
$$

The characteristic timescale $t_{\mathrm{PR}} \equiv r / v_{r, P R}$ on which the particle distance and temperature vary under the action of the PR drag is then

$$
t_{\mathrm{PR}}=\frac{8 \pi}{3} \frac{\rho a_{0} c^{2}}{L_{\star}} r^{2} \approx 10^{4} \mathrm{yr} \frac{\rho_{1} a_{0,1}}{L_{\star,-3}}\left(\frac{r}{0.2 R_{\odot}}\right)^{2} .
$$

Since $t_{\mathrm{PR}}$ is much longer than the sublimation timescale $t_{s}$ given by equation (13) it is clear that in the optically thin disks sublimation must be occurring in a quasi-static fashion: particles slowly drift inward under the action of the PR drag and their temperature steadily rises. At some radius $R_{i n}^{\text {thin }}$ their temperature reaches $T_{s}$, and particles turn into metal gas on a (short) sublimation timescale $t_{s}$. That means that the inner edge of the optically thin disk is set by the condition $T_{\text {thin }}\left(R_{i n}^{\text {thin }}\right)=T_{s}$, with $T_{s}$ given by equation (8). Thus,

$$
R_{i n}^{\text {thin }}=\frac{R_{\star}}{2}\left(\frac{T_{\star}}{T_{s}}\right)^{2} \text {. }
$$

In particular, according to Table 1 we need to take $T_{s} \approx$ $2100 \mathrm{~K}$ for olivine, which when plugged in the equation (133) yields $R_{i n} \approx 11 R_{\star}$ for $T_{\star}=10^{4} \mathrm{~K}$. This is about $60 \%$ further from the star that in the case of an optically thick disk, see $₫ 3$.

\section{APPLICATION TO OBSERVED SYSTEMS.}

We now apply ideas developed in $\$ 3$ to a sample of observed WDs with debris disks. We start by rewriting the expression (29) for $C$ as

$$
\begin{aligned}
C & =C_{\star}+C_{p}, \\
C_{\star} & \equiv \ln \left[\frac{R_{\star} T_{\star}^{2}}{\dot{M}_{Z}}\right], \quad C_{p} \equiv \ln \left[\frac{\langle\alpha\rangle K_{0} a_{0}}{2 \zeta T_{0}^{2}}\right] .
\end{aligned}
$$

Here $C_{\star}$ is a parameter, which depends only on measurable properties of the system - WD radius, effective temperature, and metal accretion rate. All parameters characterizing the particle properties $-K_{0}, a_{0}$, etc. are absorbed into $C_{p}$. Assuming a particular composition of particles and a value of particle radius $a_{0}$ fixes $C_{p}$ and allows one to obtain a theoretical relation between $T_{\text {rim }}$ and $C_{\star}$ using equations (26), (34), and (35). By looking at different particle compositions one can compare the corresponding theoretical $T_{\operatorname{rim}}\left(C_{\star}\right)$ curves with the properties of observed systems.

Such comparison requires the knowledge of $R_{\star}, T_{\star}$, $\dot{M}_{Z}$, which we take from the literature. One also needs to know $T_{\text {rim }}$ for each of the WD+disk systems, and we derive this parameter as $T_{\text {thin }}$ from equation (3), in which we use $T_{i n}$ - the innermost disk temperature inferred from the SED fitting - for $T_{\text {thick }}$. We use the values of $T_{i n}$ determined in the literature when available, and we provide our own fits otherwise. The summary of WD+disk parameters used in our comparison with theory is provided in Table 2 .
In Figure 4 we show theoretical $T_{\text {rim }}\left(C_{\star}\right)$ curves for different particle compositions. In our calculations we always assume $a_{0}=1 \mathrm{~cm}$ particles, $\langle\alpha\rangle=0.1$, and $\zeta=0.1$ (all dimensional quantities are expressed in CGS units). We also plot the locations of observed systems from Table 2 in $C_{\star}-T_{\text {rim }}$ space with hexagons.

As expected, very refractory particles made of graphite, $\mathrm{SiC}$, and $\mathrm{Al}_{2} \mathrm{O}_{3}$ are characterized by considerably higher values of theoretical $T_{\text {rim }}$ (for the same $C_{\star}$ ) than if they were to have more volatile compositions, e.g. were made of iron. The difference in $T_{\text {rim }}$ can easily exceed $10^{3} \mathrm{~K}$.

The vast majority of observed systems lies in between the two extremes determined by the iron and graphite. It is clear from this plot that the pure graphite composition is not acceptable for particles in the observed WD+disk systems - all of them are below the corresponding theoretical curve. Also, only a handful of systems lie close to the theoretical $T_{\text {rim }}\left(C_{\star}\right)$ curve for iron. The majority of observed WD+disk systems tend to gravitate towards $T_{\text {rim }}\left(C_{\star}\right)$ curves computed for CAI and olivine-like compositions. At the same time about a third of the systems in the upper right corner of the figure are consistent with more refractory compositions such as $\mathrm{SiC}$ or $\mathrm{Al}_{2} \mathrm{O}_{3}$.

When comparing characteristics of observed systems with theoretical predictions for $T_{\text {rim }}\left(C_{\star}\right)$, a couple of issues have to be kept in mind. First, observational determination of parameters of the WD+disk systems is prone to errors. This is not so serious for the determination of $T_{\star}$, which is typically quite accurate, or $R_{\star}$, which does not span a large range anyway. However, the determination of $\dot{M}_{Z}$ from the data depends on the unknown composition of the parent body that formed the disk, and may have large error bars. On the other hand, $C_{\star}$ * depends on these characteristics only logarithmically, so that even large uncertainties in these parameters would result in a relatively small horizontal shift of observational points in Figure 4

The uncertainty in measuring $T_{\text {rim }}$ is much more serious. This is because the determination of $T_{i n}$ relies on fitting the flat disk model to the SED, and $T_{i n}$ can be highly degenerate with other parameters, such as the disk inclination (Girven et al. 2012). Also, according to equation (3) $T_{\text {rim }} \propto T_{i n}^{2 / 3}$, so that the errors in determination of $T_{i n}$ from SED directly propagate into the uncertainty in $T_{\text {rim }}$. As a result, observational data points in Figure 44 can have significant vertical errorbars.

Another thing to keep in mind, is that when computing the theoretical $T_{\text {rim }}\left(C_{\star}\right)$ curves we make certain assumptions about particle properties, such as their size $a_{0}$ or accommodation coefficient $\langle\alpha\rangle$. Variation of these parameters from their adopted values affects the value of $C_{p}$ and causes horizontal shift of the $T_{\text {rim }}\left(C_{\star}\right)$ curves. For example, increasing the value of accommodation coefficient $\langle\alpha\rangle$ from 0.1 to 1 displaces the theoretical curves to the left by $\Delta C_{\star}=2.3$. This would put observational data points in better agreement with the more refractory particle compositions.

\section{DISCUSSION.}

The physical model for the inner rim structure in the optically thick case presented in $\$ 3$ naturally allows us to explain the high inner disk temperatures $T_{i n}$ inferred 
Table 2

Properties of disk-hosting WDs used in this work

\begin{tabular}{|c|c|c|c|c|c|c|c|c|c|c|}
\hline Name & SpT & $\begin{array}{l}M_{\star} \\
M_{\odot}\end{array}$ & $\begin{array}{l}R_{\star} \\
R_{\odot}\end{array}$ & $\begin{array}{c}T_{\star} \\
\mathrm{K}\end{array}$ & $\begin{array}{c}\log _{10} \dot{M} / \mathrm{s} \\
\mathrm{g}\end{array}$ & $\begin{array}{l}\text { Gas Disk } \\
\text { Detected }\end{array}$ & $\begin{array}{c}T_{i n} \\
\mathrm{~K}\end{array}$ & $\underset{\mathrm{K}}{T_{\mathrm{rim}}}$ & $\begin{array}{c}C_{\star} \\
\mathrm{CGS}\end{array}$ & Ref. \\
\hline GD 16 & DAZB & 0.59 & 0.014 & 11500 & 8.0 & & $1300^{\mathrm{a}}$ & 2460 & 21.0 & 1 \\
\hline GD 133 & DAZ & 0.59 & 0.014 & 12200 & 8.5 & & 1200 & 2380 & 19.9 & 1,2 \\
\hline GD 40 & DBZ & 0.59 & 0.013 & 15200 & 9.9 & & 1200 & 2560 & 17.1 & 1 \\
\hline GD 56 & DAZ & 0.60 & 0.015 & 14200 & 8.5 & & 1700 & 3160 & 20.3 & 1,2 \\
\hline $\mathrm{J} 1228+1040$ & DAZ & 0.77 & 0.011 & 22020 & 9.3 & yes & 1670 & 3610 & 19.0 & 3,4 \\
\hline PG1015+161 & DAZ & 0.61 & 0.014 & 19300 & 9.3 & & 1200 & 2770 & 19.0 & 1,2 \\
\hline Ton345 & DBZ & 0.70 & 0.010 & 18600 & 9.4 & yes & 1500 & 3180 & 18.4 & $5,6,7$ \\
\hline SDSS1043+0855 & $\mathrm{DAZ}$ & 0.66 & 0.012 & 17900 & 9.0 & yes & 1400 & 3000 & 19.4 & 8 \\
\hline G29-38 & DAZ & 0.62 & 0.013 & 11700 & 8.7 & & 1200 & 2350 & 19.3 & $1,9,10$ \\
\hline GD 362 & DAZB & 0.73 & 0.013 & 10500 & 10.4 & & 1200 & 2260 & 15.2 & $1,9,11$ \\
\hline SDSS0959 & DAZ & 0.64 & 0.012 & 13280 & 7.9 & & 1600 & 2970 & 21.3 & 12 \\
\hline SDSS1221 & DAZ & 0.73 & 0.011 & 12250 & 7.7 & & 1400 & 2640 & 21.6 & 12 \\
\hline SDSS1557 & $\mathrm{DAZ}$ & 0.42 & 0.018 & 22810 & 8.8 & & 1400 & 3250 & 20.8 & 12 \\
\hline GD 61 & DBZ & 0.71 & 0.011 & 17280 & 8.81 & & 1300 & 2820 & 19.7 & 13,14 \\
\hline J0738+1835 & DBZ & 0.84 & 0.010 & 13950 & 11.11 & yes & 1600 & 3020 & 13.9 & 16 \\
\hline HE 0110-5630 & DBAZ & 0.71 & 0.012 & 19200 & 8.4 & & 1000 & 2450 & 20.9 & 17,18 \\
\hline HE 1349-2305 & DBAZ & 0.67 & 0.012 & 18200 & 8.7 & & 1700 & 3430 & 20.1 & 17,18 \\
\hline
\end{tabular}

a These numbers were calculated in this paper.

b (1) Farihi et al. 2009; (2) Jura et al. 2007a; (3) Brinkworth et al. 2009; (4) Gänsicke et al. 2006; (5) Farihi et al. 2010; (6) Melis et al. 2010; (7) Gänsicke et al. 2008; (8) Brinkworth et al. 2012; (9) Farihi et al. 2008; (10) Zuckerman et al. 2003; (11) Zuckerman et al. 2007; (12) Farihi et al. 2012; (13) Farihi et al. 2011; (14) Jura \& Xu 2012; (15) Kilic et al. 2012; (16) Dufour et al. 2012; (17) Girven et al. 2012; (18) Koester et al.|2005.

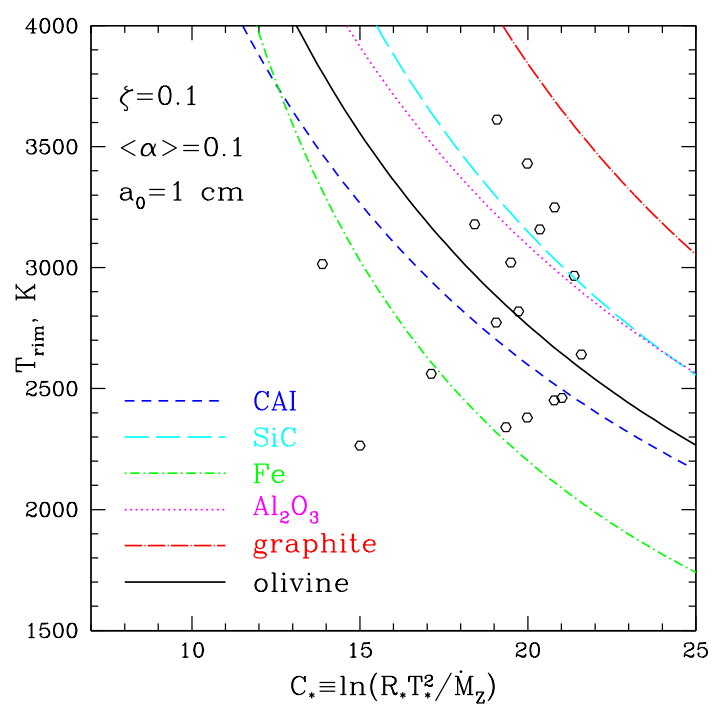

Figure 4. Comparison of observed WD+disk properties with theoretical predictions in $C_{\star}-T_{\text {rim }}$ space $\left(C_{\star}\right.$ is defined by equation (35)). Theoretical $T_{\text {rim }}\left(C_{\star}\right)$ curves computed for different particle compositions are labeled on the plot. Their calculation assumes $a_{0}=1 \mathrm{~cm}$ particles, $\zeta=0.1$, and $\langle\alpha\rangle=0.1$. Note that most of the observed systems are consistent with particles being made of Si-bearing materials, such as olivine or CAI.

from the SEDs of debris disks around some WDs. The existence of a narrow inner rim of the disk heated to a temperature $T_{\text {rim }}$ above the quasi-static sublimation temperature $T_{s}$ (see equation (8) ) is the key ingredient of the model.

The radial width of the inner $\operatorname{rim} L$ can be estimated by multiplying the time to cross it $t_{\text {cross }}$ by the velocity $v_{r, P R}$, given by equations (24) and (31) correspondingly:

$$
L \sim 10 \mathrm{~cm} \frac{a_{0,1} R_{\star,-2} L_{\star,-3}}{\zeta_{0.1} \dot{M}_{Z, 8}}\left(\frac{0.2 \mathrm{AU}}{R_{\text {in }}}\right)
$$

Thus, one typically finds the width of the inner rim to be $\sim 10$ particle radii.

Note that the radial speed of particles in massive disks can be affected by aerodynamic coupling between the particulate and gaseous disks, and in consequence deviate from $v_{r, P R}$. Nevertheless, equation (36) serves as a reasonable order of magnitude estimate of $L$ and clearly demonstrates that $L \ll R_{\text {in }}$. As a result, the contribution of the hot inner rim to the SED of the debris disk is completely negligible, and its spectrum is determined only by emission from the parts of the disk located behind the rim.

Our results in \$3, 4 allow us to address the differences in spectra of disks with optically thick or thin inner regions. In Figure 5 we show several spectra produced by disks around a $T_{\star}=10^{4} \mathrm{~K}, R_{\star}=0.01 R_{\odot}$ WD located $10 \mathrm{pc}$ away from us, and inclined with respect to our line of sight with $\cos i=0.5$. The model, which is optically thick everywhere, has constant optical depth $\tau=10$ and extends from the outer radius $R_{\text {out }}=R_{\odot}$ to $R_{i n}^{\text {thick }} \approx 7 R_{\star}$ given by equation (15). Models with optically thin tails also have constant optical depth $\tau=10$ between $R_{\text {out }}=$ $R_{\odot}$ and some intermediate radius $R_{1}$, which is different for each model. Inside of $R_{1}$ we assume an optically thin tail with constant $\tau=\zeta\left(R_{1}\right)$ (or $\tau_{\|}=r / R_{1}$ ) to extend from $R_{1}$ down to $R_{i n}^{\text {thin }} \approx 11 R_{\star}$ given by equation (33). This is the characteristic distribution of $\tau$ in the inner optically thin tails of the disks evolving under the action of the PR drag, see Bochkarev \& Rafikov (2011).

One can see that the disk which is optically thick everywhere produces more flux. This is expected because disks with optically thin tails do not extend as far inward, and are inefficient at absorbing and re-radiating in regions interior to $r=R_{1}$. The spectral shape is also different, in part because particles in the optically thin tail are hotter than particles in the optically thick tail at the same radius. This may allow one to diagnose the 


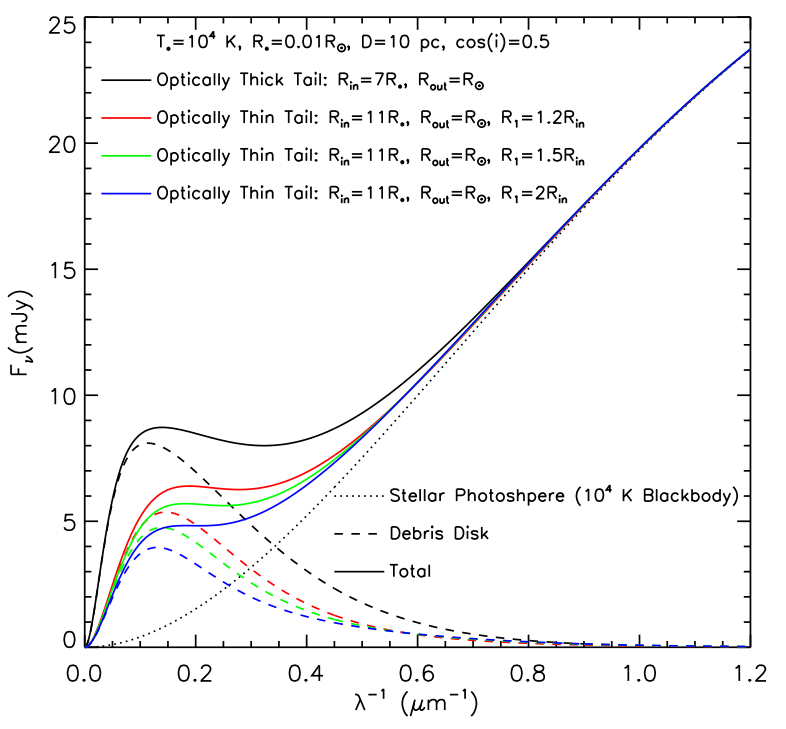

Figure 5. Spectra of debris disks with optically thick and optically thin inner regions. All models feature optically thick regions with $\tau=10$, which extend from $R_{\text {out }}=R_{\odot}$ to $R_{i n}^{\text {thick }}$ in the optically thick case and to $r=R_{1}$ (indicated in the panel) in the models with optically thin tails. Inside $R_{1}$ tails have $\tau=\zeta\left(R_{1}\right) \ll 1$. Stellar parameters are indicated on the panel. See text for more details.

presence of an inner optically thin tail using just the disk SED. Such optically thin tails may be expected in systems characterized by $\dot{M}_{Z} \sim 10^{8} \mathrm{~g} \mathrm{~s}^{-1}$, i.e. close to the value provided by PR drag alone. In the runaway scenario of Metzger et al. (2012), one expects systems with higher $\dot{M}_{Z}$ to be evolving due to aerodynamic coupling with the gaseous disk, in which case the disk is optically thick all the way down to $R_{i n}^{\text {thick }}$.

Comparison of our theory with characteristics of observed WD+debris disk systems shows that in general (barring the uncertainties related to measurement errors and poorly constrained modeling parameters) properties of these systems are consistent with Si-rich particle composition. In other words, we find that CAI- or olivinelike compositions of particles are in reasonable agreement with the locations of the inner rims in the majority of observed disk-hosting systems.

This result reinforces previous conclusions about the Si-rich nature of the accreted material based on different and independent lines of evidence. In particular (and most importantly), direct measurements of the metal abundances in the WD atmospheres show that the composition of accreted material is consistent with that of the inner Solar System bodies, which are known to be Si-rich (Zuckerman et al. 2007; Klein et al. 2010, 2011; Jura et al. 2012). These measurements also demonstrate the accreted bodies to be carbon-poor (Jura|2006), which is again consistent with our results - essentially none of the observed WD+disk systems lie close to the C-based curve in Figure 4. Additional evidence in favor of Si-rich particle composition comes from the measurement of 10$\mu \mathrm{m}$ bump in debris disk spectra obtained with Spitzer IRS (Jura et al. 2007b, 2009). This feature is usually interpreted as being produced by the $\mu \mathrm{m}$-size silicate particles.

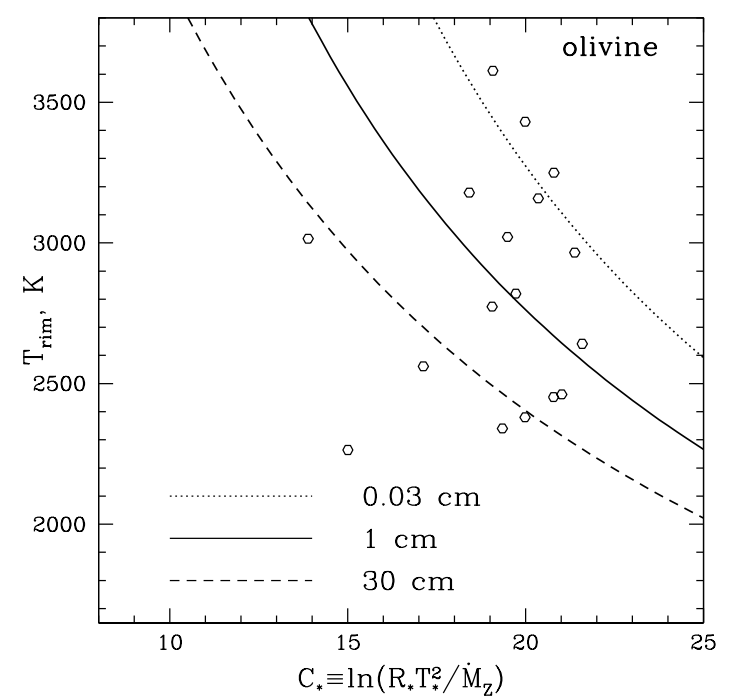

Figure 6. Effect of varying the particle radius $a$ on the theoretical $T_{\text {rim }}-C_{\star}$ dependence for olivine and comparison with observational data.

Using these independent lines of evidence supporting the Si-rich nature of the debris disk constituents we may approach our findings from a different perspective. In particular, by postulating disk particles to be Si-rich we can put constraint on their sizes. Results presented in Figure 4 do a reasonably good job at reproducing characteristics of observed systems by assuming $a=1 \mathrm{~cm}$ particles. Varying $a$ would displace the theoretical curves horizontally and they would remain consistent with observations only within a certain range of particle sizes. Figure 6 illustrates this variation of the $T_{\text {rim }}-C_{\star}$ relation; one can easily infer from it that particle sizes should lie within the range $a=0.03-30 \mathrm{~cm}$. Otherwise the properties of the inner disk rims in the majority of the observed systems will not be consistent with our theoretical calculations.

Interestingly, this range of particle sizes is consistent with other indirect measurements of $a$ reported in the literature. In particular, Graham et al. (1990) found $a \lesssim 10 \mathrm{~cm}$ based on the variability of the reprocessed IR emission of disk particles. Metzger et al. (2012) found $a \lesssim$ several $\mathrm{cm}$ to provide the best fit to the runaway picture of the disk evolution. Finally, Saturn rings, which are thought to be rather close in properties to circumWD debris disks, are also predominantly composed of $1-100 \mathrm{~cm}$ particles (Cuzzi et al. 2010).

Our model naturally explains the presence of solid particles even around hot WDs, with $T_{\star} \approx 20,000 \mathrm{~K}$, e.g. J1228+1040 and SDSS1557. Conventional theory finds it difficult to account for such systems. Indeed, equation (1) predicts that around $T_{\star}=20,000 \mathrm{~K}, R_{\star}=0.015 R_{\odot}$ WD directly illuminated particles must have a temperature of $1700 \mathrm{~K}$ at the tidal radius of $\sim R_{\odot}$. This is significantly higher than the sublimation temperature of $1300-1500 \mathrm{~K}$ usually assumed based on protoplanetary disk studies (Lodders 2003). Our calculations first show that in fact the sublimation temperature $T_{s}$ can easily be higher than $1700 \mathrm{~K}$, see Table 1, which guarantees the survival of even the optically thin disks with directly exposed particles within tidal radii of hot WDs. Second, 
in the optically thick case, shielding of the disk by the inner rim particles allows $R_{\text {in }}$ to be as small as $0.3 R_{\odot}$ (for $R_{\star}=10^{-2} R_{\odot}$, and keeping all other parameters equal to their values in equation (29)).

Our present calculations were designed to demonstrate the main qualitative features of the inner rim structure and thus made a number of simplifying assumptions. One of them is the single size of particles in the disk, while in reality a distribution of particle sizes should be present. We expect that in this case the value of $a$ in the definition (35) of $C_{p}$ would be replaced with some properly weighted average of the particle size distribution, but the main results would not change.

Another simplification is the assumed single chemical composition of all particles. If the disk contains particles of different compositions, with different $K_{0}$ and $T_{0}$, then one may expect a "multi-rim" structure to form, in which different chemical species sublimate at different radii. In this case the inner radius of the disk would be determined by the properties of the most refractory particles in the disk that survive at the closest separation from the WD. Observations of the inner disk properties (i.e. $T_{i n}$ ) would then be sensitive to characteristics of only this particular particle population (as long as the disk is optically thick everywhere).

\section{SUMMARY.}

We explored the structure of the inner parts of compact debris disks around WDs with the goal of resolving the "inner rim puzzle" — the difficulty with reconciling the high inner disk temperatures inferred from the SED with the material properties of putative constituent particles. We first show that because of the much higher vapor pressure of metals in these hydrogenpoor disks compared to the hydrogen-rich protoplanetary disks, the quasi-static sublimation temperature $T_{s}$ of different species in circum-WD disks is typically 300-400 $\mathrm{K}$ higher than in their conventional protoplanetary analogues. This revised value of $T_{s}$ determines the (smaller than was thought before) value of the inner radius for the optically thin disks, given by equations (8) \& (33).

We demonstrate that optically thick circum-WD disks feature narrow inner rims, which are superheated above $T_{s}$. This allows inner disk radii in such systems, described by equations (15), (26), \& (27), to lie quite close to the WD, easily at separations $\sim 10 R_{\star}$. The main physical ingredients needed for the existence of such superheated inner rim are (1) accretion of particles through the disk, which can be easily maintained at the necessary level by Poynting-Robertson drag, (2) shielding of particles behind the rim from starlight by the rim particles, and (3) dynamic nature of the sublimation process inside the hot rim. The combination of these ingredients naturally allows particles to reach temperatures of order $1600-1700 \mathrm{~K}$ just behind the rim, which is needed to explain the SEDs of some systems. Particles inside the rim are heated to $2500-3500 \mathrm{~K}$ and undergo rapid sublimation as they migrate in. Using this model we can naturally explain the existence of particulate debris disks even around hot WDs, with effective temperature $\gtrsim 20,000 \mathrm{~K}$.

We compare our predictions with existing observations of the WD+disk systems. We find that properties of particles in debris disks are consistent with Si-rich compo- sition, such as olivine or CAI-like material. Very refractory (such as graphite) or more volatile (such as iron) compositions are clearly disfavored by this comparison. Assuming that circum-WD disks are indeed composed of Si-rich particles we constrain typical particle size to lie roughly between 0.03 and $30 \mathrm{~cm}$, in agreement with other indirect evidence for cm-size objects in such disks.

The authors thank Bruce Draine and Michael Jura for stimulating discussions. The financial support for this work is provided by the Sloan Foundation and NASA via grant NNX08AH87G.

\section{REFERENCES}

Alcock, C., Fristrom, C. C., \& Siegelman, R. 1986, ApJ, 302, 462 Bochkarev, K. V., \& Rafikov, R. R. 2011, ApJ, 741, 36

Brewer, L., \& Searcy, A. W. 1951, Journal of the American Chemical Society, 73, 5308

Brinkworth, C. S., Gänsicke, B. T., Girven, J. M., et al. 2012, ApJ, 750, 86

Brinkworth, C. S., Gänsicke, B. T., Marsh, T. R., Hoard, D. W., \& Tappert, C. 2009, ApJ, 696, 1402

Chase, M. W. J., Davies, C. A., Downey, J. R. J., et al. 1985, J. Phys. Chem. Ref. Data, 14, Suppl. No. 1

Chiang, E. I., \& Goldreich, P. 1997, ApJ, 490, 368

Cuzzi, J. N., Burns, J. A., Charnoz, S., et al. 2010, Science, 327, 1470

Debes, J. H., \& Sigurdsson, S. 2002, ApJ, 572, 556

Dufour, P., Kilic, M., Fontaine, G., et al. 2012, ApJ, 749, 6

Farihi, J. 2011, in American Institute of Physics Conference Series, Vol. 1331, American Institute of Physics Conference Series, ed. S. Schuh, H. Drechsel, \& U. Heber, 193-210

Farihi, J., Brinkworth, C. S., Gänsicke, B. T., et al. 2011, ApJl, $728, \mathrm{~L} 8$

Farihi, J., Gänsicke, B. T., Steele, P. R., et al. 2012, MNRAS, 421, 1635

Farihi, J., Jura, M., Lee, J.-E., \& Zuckerman, B. 2010, ApJ, 714, 1386

Farihi, J., Jura, M., \& Zuckerman, B. 2009, ApJ, 694, 805

Farihi, J., Zuckerman, B., \& Becklin, E. E. 2008, ApJ, 674, 431

Friedjung, M. 1985, A\&A, 146, 366

Gänsicke, B. T., Koester, D., Marsh, T. R., Rebassa-Mansergas, A., \& Southworth, J. 2008, MNRAS, 391, L103

Gänsicke, B. T., Marsh, T. R., \& Southworth, J. 2007, MNRAS, 380, L35

Gänsicke, B. T., Marsh, T. R., Southworth, J., \&

Rebassa-Mansergas, A. 2006, Science, 314, 1908

Girven, J., Brinkworth, C. S., Farihi, J., et al. 2012, ApJ, 749, 154

Graham, J. R., Matthews, K., Neugebauer, G., \& Soifer, B. T. 1990, ApJ, 357, 216

Guhathakurta, P., \& Draine, B. T. 1989, ApJ, 345, 230

Jura, M. 2003a, ApJ, 582, 1032

-. 2003b, ApJl, 584, L91

-. 2006, ApJ, 653, 613

Jura, M., Farihi, J., \& Zuckerman, B. 2007a, ApJ, 663, 1285

-. 2009, AJ, 137, 3191

Jura, M., Farihi, J., Zuckerman, B., \& Becklin, E. E. 2007b, AJ, 133,1927

Jura, M., \& Xu, S. 2012, AJ, 143, 6

Jura, M., Xu, S., Klein, B., Koester, D., \& Zuckerman, B. 2012, ApJ, 750, 69

Kilic, M., Patterson, A. J., Barber, S., Leggett, S. K., \& Dufour, P. 2012, MNRAS, 419, L59

Kilic, M., von Hippel, T., Leggett, S. K., \& Winget, D. E. 2005, ApJl, 632, L115

-. 2006, ApJ, 646, 474

Kimura, H., Mann, I., Biesecker, D. A., \& Jessberger, E. K. 2002, Icarus, 159,529

Klein, B., Jura, M., Koester, D., \& Zuckerman, B. 2011, ApJ, 741,64

Klein, B., Jura, M., Koester, D., Zuckerman, B., \& Melis, C. 2010, ApJ, 709, 950

Koester, D., Rollenhagen, K., Napiwotzki, R., et al. 2005, A\&A, 432,1025

Lodders, K. 2003, ApJ, 591, 1220

Melis, C., Jura, M., Albert, L., Klein, B., \& Zuckerman, B. 2010, ApJ, 722, 1078

Metzger, B. D., Rafikov, R. R., \& Bochkarev, K. V. 2012 MNRAS, 423, 505 
Podolak, M. 2010, in IAU Symposium, Vol. 263, IAU Symposium, ed. J. A. Fernández, D. Lazzaro, D. Prialnik, \& R. Schulz, $19-28$

Rafikov, R. R. 2011a, ApJl, 732, L3

-. 2011b, MNRAS, 416, L55

Richter, F. M., Janney, P. E., Mendybaev, R. A., Davis, A. M., \&

Wadhwa, M. 2007, Geochimica et Cosmochimica Acta, 71, 5544

Zatisev, A. I., Shelkova, N. E., Litvina, A. D., et al. 2001, High

Temperature, 39, 388
Zuckerman, B., \& Becklin, E. E. 1987, Nature, 330, 138 Zuckerman, B., Koester, D., Melis, C., Hansen, B. M., \& Jura, M. 2007, ApJ, 671, 872

Zuckerman, B., Koester, D., Reid, I. N., \& Hünsch, M. 2003, ApJ, 596,477

\section{APPENDIX}

\section{THERMAL BALANCE IN THE DISK OF SOLIDS.}

Grains in the inner rim are being heated and cooled by four processes: (1) heating by starlight, (2) heating by gas, (2) cooling by thermal radiation from particle surfaces, and (4) removal of thermal energy by sublimating atoms/molecules. All these processes scale linearly with the surface area of the particles. As a result, the temperature of grains directly exposed to starlight (assuming full absorption of the incoming radiation) is implicitly given as a function of the distance from the WD by the following formula (Podolak 2010):

$$
\begin{aligned}
& \frac{1}{4} \sigma T_{\star}^{4}\left(\frac{R_{\star}}{r}\right)^{2}+Q_{\text {gas }}=\sigma T^{4}+Q_{\mathrm{sub}}, \\
& Q_{\mathrm{sub}}=\dot{m}(T) L_{\mathrm{sub}}, \quad Q_{\mathrm{gas}}=\frac{\rho_{g} c_{\mathrm{s}}}{2 \mu} \varepsilon k_{B}\left(T_{\mathrm{gas}}-T\right),
\end{aligned}
$$

where $L_{\mathrm{sub}}$ is the specific heat of sublimation of the particle material, $\varepsilon$ is the efficiency of heat exchange between gas and particles, and $\rho_{g}$ is the gas density.

Using equation (10) we can estimate $\Sigma_{g} \approx \dot{M}_{Z} /(3 \pi \nu)$ so that

$$
\rho_{g}=\frac{\Sigma_{g} \Omega}{c_{s}}=\frac{\dot{M}_{Z} \Omega^{2}}{3 \pi \alpha_{\nu} c_{s}^{3}} .
$$

This allows us to compare the contribution of gas heating $Q_{\text {gas }}$ with stellar irradiation $Q_{\star}$ (first term in the left hand side of equation (A1)):

$$
\frac{Q_{\mathrm{gas}}}{Q_{\star}} \approx \frac{2 \varepsilon}{3 \pi} \frac{G M_{\star} \dot{M}_{Z}}{\alpha_{\nu} r R_{\star}^{2} \sigma T_{\star}^{4}}\left(1-\frac{T}{T_{\mathrm{gas}}}\right) \sim 10^{-4} \frac{\varepsilon \dot{M}_{Z, 8} M_{\star, 1}}{\alpha_{\nu,-2} T_{\star, 4}^{4} R_{\star,-2}^{2}}\left(\frac{r}{0.2 R_{\odot}}\right)^{-1} .
$$

Therefore, gas heating is typically unimportant for the thermal balance of particles compared to heating by starlight, in contrast to the conclusion reached by Jura et al. (2007b), who looked at conduction in gas phase as the means to lower particle temperature. This difference is predominantly caused by the high gas density $\left(\sim 10^{2}\right.$ times higher than in equation (A3) ) used in Jura et al. (2007b).

Using prescription (7) with $\langle\alpha\rangle=0.1$ and the typical (for olivine) value of $L_{\text {sub }}=3.2 \times 10^{10} \mathrm{erg} \mathrm{g}^{-1}$ from Kimura et al. (2002) we can also estimate the relative contribution of sublimation to the cooling of particles by evaluating $Q_{\mathrm{sub}} / \sigma T^{4}$. We find this ratio to be about unity for particles heated to $\approx 3400 \mathrm{~K}$. At $T=3000 \mathrm{~K}$ the ratio of the energy loss by sublimation to radiative cooling rate is about 0.1 . Thus, for WD+disk systems with $T_{\text {rim }} \lesssim 3000 \mathrm{~K}$ one can safely neglect $Q_{\text {sub }}$ in equation (A1). Then the thermal balance everywhere in the disk is determined by the equilibrium between stellar heating and radiative cooling only, which provides justification for using equations (11) and (2) in this work. This assumption is good for the majority of observed systems shown in Figure 4, and even for a handful of systems with $T_{\text {rim }} \approx 3000-3500$ our theoretical curves should still be at least qualitatively correct.

\section{DATA ON THE MASS SUBLIMATION RATES}

Here we present the details on the derivation of mass sublimation rates for different elements shown in Table 1.

Olivines Calculation of the vapor pressure for the olivine-like silicate grains (e.g. $\mathrm{Mg}_{2} \mathrm{SiO}_{4}$ ) is complicated due to the fact that these molecules do not exist in the gas phase. Nevertheless, Guhathakurta \& Draine (1989) suggest the following expression for the (number) rate of Si sublimation from the olivine surface: $R_{\mathrm{Si}} \approx 7 \times 10^{30}\langle\alpha\rangle \exp (-68,100 / T)$ $\mathrm{cm}^{-2} \mathrm{~s}^{-1}$. The mass sublimation rate of olivine is then given by $\mu_{\mathrm{oli}} R_{\mathrm{Si}}$, where $\mu_{\text {oli }}=141 \mathrm{~m}_{p}$ is the mean molecular weight of $\mathrm{Mg}_{2} \mathrm{SiO}_{4}$.

Graphite For pure graphite Guhathakurta \& Draine (1989) give the (number) rate of C sublimation from the graphite surface of $R_{\mathrm{C}} \approx 4.6 \times 10^{30}\langle\alpha\rangle \exp (-81,200 / T)$, which then allows us to calculate $K_{0}$ from the mass sublimation rate $R_{\mathrm{C}} \mu_{\mathrm{C}}$, where $\mu_{\mathrm{C}}=12 m_{p}$ is the mean molecular weight of carbon.

CAI Richter et al. (2007) consider evaporation of CAI-like liquids and come up with the following (number) rate of Si escaping a CAI-like surface: $R_{\mathrm{Si}} \approx 2.5 \times 10^{31}\langle\alpha\rangle \exp (-69,400 / T)$. Using gehlenite $\left(\mathrm{Ca}_{2} \mathrm{Al}_{2} \mathrm{SiO}_{7}\right)$ as a typical CAI-like material (mean molecular weight $274 m_{p}$ ) we obtain sublimation parameters indicated in Table 1.

Iron Zatisev et al. (2001) provide the data on the vapor pressure of Fe: $P_{\text {vap }}^{\mathrm{Fe}}=2.8 \times 10^{11} \exp (-45,400 / T) \mathrm{Pa}$ for $T \approx 1800-1900 \mathrm{~K}$. From these data we determine the mass sublimation rate according to the formula $\dot{m}_{\mathrm{Fe}}=$ 
$\langle\alpha\rangle P_{\text {vap }}^{\mathrm{Fe}}\left(\mu_{\mathrm{Fe}} / 2 \pi k_{B} T\right)^{1 / 2}$, where $\mu_{\mathrm{Fe}} \approx 56 m_{p}$ and we take $T=1600 \mathrm{~K}$ with the expectation that the thermophysical parameters of Fe remain roughly the same at this temperature.

SiC Using the thermophysical data presented in Chase et al. (1985) we derive the following fit to the behavior of the vapor pressure of $\mathrm{Si}$ above the $\mathrm{SiC}$ surface in the range $T=1800-3000 \mathrm{~K}: P_{\text {vap }}^{\mathrm{Si}}=9 \times 10^{13} \exp (-73,700 / T)$ dyne $\mathrm{cm}^{-2}$. Since the surface loses $\mathrm{C}$ atoms in addition to $\mathrm{Si}$ we evaluate $\mathrm{SiC}$ mass loss rate as $\dot{m}_{\mathrm{SiC}}=$ $\langle\alpha\rangle P_{\mathrm{vap}}^{\mathrm{Si}}\left(\mu_{\mathrm{SiC}} / \mu_{\mathrm{Si}}\right)\left(\mu_{\mathrm{Si}} / 2 \pi k_{B} T\right)^{1 / 2}$, where $\mu_{\mathrm{SiC}}=40 m_{p}, \mu_{\mathrm{Si}}=28 m_{p}$ and we take $T=2400 \mathrm{~K}$.

$\mathbf{A l}_{2} \mathbf{O}_{3}$ For pure corundum $\left(\mathrm{Al}_{2} \mathrm{O}_{3}\right)$ the basic reaction which is thermodynamically most likely is $\mathrm{Al}_{2} \mathrm{O}_{3} \rightarrow 2 \mathrm{AlO}+\mathrm{O}$ (a different reaction dominates for $\mathrm{Al}-\mathrm{Al}_{2} \mathrm{O}_{3}$ mixture, see Brewer \& Searcy 1951). Using the data in Chase et al. (1985) we find that the vapor pressure of $\mathrm{O}$ above the corundum surface is $P_{\text {vap }}^{O}=3.6 \times 10^{14} \exp (-80,500 / T) \mathrm{dyne} \mathrm{cm}^{-2}$ for $T=1800-3000 \mathrm{~K}$. Accounting for the mass of Al leaving the surface together with $\mathrm{O}$ we arrive at the sublimation characteristics of corundum presented in Table 1 . 